Results 694 patients (334 male, 340 female), median age 67 (range $21-99), 77 \%$ patients over 50 . Endoscopy was visually normal in $45 \%$, the commonest visual abnormalities were Oesophagitis (18\%) and malignancy (13\%). 23\% of patients had biopsies. Suspected malignancy or BO were most likely to have biopsies taken (65\% and $64 \%$ respectively), $9 \%$ visually normal endoscopies were biopsied. $83 \%$ (49 cases) with suspected malignancy had histological correlation, 17\% (11 cases) had BO or Oesophagitis. Three patients were found to have malignancy where the visual diagnosis had been Oesophagitis or benign stricture. Both $\mathrm{BO}$ and Oesophagitis had $>80 \%$ correlation visually and histologically. Six cases of EO were found, all were visually normal. One suspected case was seen at endoscopy, this was histologically normal.

Conclusion There was generally good correlation between visual and endoscopic diagnosis, particularly in malignancy, however biopsy number was lower than expected. Failure to biopsy may lead to missed diagnosis of cancer or dysplasia. All cases of EO in adults had normal endoscopy, few patients with dysphagia and normal endoscopy had biopsies taken. EO may be commoner than suspected, true rates are unknown and a high index of suspicion is needed. We should carry out more endoscopies on younger patients with symptoms in keeping with EO and biopsy normal oesophagus in cases where this diagnosis is suspected.

Competing interests None declared.

\section{PTU-222 A COMPARISON OF PATIENT TOLERANCE OF BOWEL PREPARATION REGIMENS USED FOR CONVENTIONAL COLONOSCOPY, SMALL BOWEL AND COLON CAPSULE ENDOSCOPY}

doi:10.1136/gutjnl-2012-302514c.222

K Drew, ${ }^{*}$ S Hardcastle, A J Lobo, D S Sanders, R Sidhu, M McAlindon. Gastroenterology, Sheffield Teaching Hospitals NHS Trust, Sheffield, UK

Introduction That patients tolerate swallowing a capsule better than undergoing conventional colonoscopy (CC) is self evident, but without the ability to cleanse the bowel during the procedure capsule endoscopy is critically reliant on a clean bowel and preparation regimens tend to be more rigorous, which may affect the patients' acceptance of the procedure. In this study, patient tolerance of a standard regimen used for colon capsule endoscopy (CCE) was compared with that used for CC and a smaller volume regimen used for small bowel capsule endoscopy (SBCE) as a control.

Methods All patients undergoing CC, SBCE and CCE on the Clinical Investigation Unit were asked to score symptoms of nausea, vomiting, bloating, abdominal pain and headache as none, mild, moderate, severe or extreme and provide an overall tolerance score on a visual analogue scale (0: intolerable; 10: no symptoms). Laxatives administered were 4L (CC) and 2L (SBCE) Klean prep (Norgine Ltd., UK). 2L Klean prep was given the day before aswell as the day of CCE when two further "booster" doses of Fleet phospho-soda (Fleet Labs., Lynchburg, USA; 30 and $25 \mathrm{ml}$ ) were also administered. $\chi^{2}$ test was used to compare patients suffering none-mild and moderate-extreme symptoms with different regimens and one-tailed t test to compare overall tolerance.

Results 104 patients had bowel preparation for CC ( $n=28)$, SBCE $(n=54)$ and CCE $(n=22)$ and suffered moderate-extreme nausea (4, 21 and $18 \%$ respectively), vomiting (4, 8, $0 \%$ ), bloating $(21,17,36 \%)$, abdominal pain $(14,25,23 \%)$ and headache $(11,21,32 \%)$. Moderate to extreme nausea was more common in those taking bowel preparation for SBCE than CC ( $p=0.04)$, but there were no differences in the distribution of any of the other symptoms between the different regimens. Overall tolerance score was (median (range)) 7.2 (1-9) with no difference between regimens. CCE and SBCE groups were similar in terms of sex (64 and 58\% female respectively) and age (mean 39 and 49 years, $\mathrm{p}=0.1$ ).

Conclusion Bowel preparation for endoscopic procedures is commonly associated with a wide range of symptoms. However patient tolerance of regimens used for CCE and CC were equivalent and indeed not demonstrably worse than the low volume preparation used for SBCE. This study suggests that the bowel preparation regimen is unlikely to influence patients' choice of CCE or CC as an investigative modality.

Competing interests None declared.

\section{PTU-223 CARDON DIOXIDE INSUFFLATION DURING ENDOSCOPIC RETROGRADE CHOLANGIOPANCREATOGRAPHY MAY REDUCE ABDOMINAL PAIN BUT IT DOES NOT ALTER THE SEDATION AND ANALGESIA REOUIREMENTS}

doi:10.1136/gutjnl-2012-302514c.223

${ }^{1} \mathrm{M}$ Bhalme, ${ }^{*} \mathrm{~F}$ Kidd, ${ }^{3} \mathrm{~J}$ Ramesh, ${ }^{4} \mathrm{D}$ Martin. ${ }^{1}$ North Manchester General Hospital, Manchester, UK; ${ }^{2}$ St Ann's Hospice, Manchester, UK; ${ }^{3}$ University of Alabama, Birmingham, USA; ${ }^{4}$ University Hospital of South Manchester, Manchester, UK

Introduction Compared to air, carbon dioxide (CO2) insufflation during endoscopic retrograde cholagiopancreatography (ERCP) may reduce bowel distension and resulting pain. However, its effect on sedation is unknown. Our objective was to investigate the effect of CO2 insufflation on the amount of sedation, analgesia and antispasmodic needed during ERCP. Secondarily, the perceived patient discomfort and complications were also examined.

Methods Using a database, we retrospectively identified 60 patients (pts) each, before and after introduction of $\mathrm{CO} 2$ insufflation for ERCP. All procedures were performed using titrating doses of intravenous fentanyl and midazolam combination aiming for a Bispectral Index (BIS) value of 85, which indicates an adequate level of deep sedation. Post ERCP abdominal pain and conscious level was assessed by experienced recovery nurses using a visual analogue scale (VAS) of 0 to 10 and AVPU (Alert, Verbal, Pain, Unresponsive) scale respectively. The statistical analysis for drug doses was carried out using Mann-Whitney U test.

Results Patient demographics such as age, sex, co-morbidities, indications and ASA grades were similar in both groups. The same median dose of intravenous hyoscine butylbromide $(20 \mathrm{mg}, \mathrm{p}=0.89$ ) and fentanyl $(75 \mu \mathrm{g}, \mathrm{p}=0.70)$ was used in both groups while the median dose of midazolam was 4.5 vs $4 \mathrm{mg}(\mathrm{p}=0.25)$ for the air and $\mathrm{CO} 2$ group respectively. The duration of procedure was 33 vs $29 \mathrm{~min}$ $(p=0.63)$ for the air and CO2 group respectively. During the first hour post procedure, the AVPU score for air group was A-45, V-12, P$2, \mathrm{U}-1$ and for CO2 group it was A-47, V-13, P-0, U-0 respectively. The incidence of abdominal pain during the first hour post procedure for air and $\mathrm{CO} 2$ groups was $10 \%$ and $0 \%(p=0.027)$ respectively, while the mean score for pain on VAS in the air group was 2 (range 1-6; $p=0.012$, Mann-Whitney $U$ test). Complications included pancreatitis ( $0 \%$ vs $1.3 \%$ ) and post-sphincterotomy haemorrhage ( $2.6 \%$ vs $0 \%$; $p=0.5$, Fisher's Exact test) in the air and $\mathrm{CO} 2$ groups respectively. All complications settled with conservative management. No serious cardio-pulmonary complication was noted in either group.

Conclusion Carbon dioxide insufflation during ERCP reduces the incidence and severity of post procedure abdominal pain based on VAS but it does not influence the amount of sedation or analgesia required to achieve sufficient palliation of pain during the procedure The use of $\mathrm{CO} 2$ in unselected well sedated, prone patients appears to be safe.

Competing interests None declared. 